\title{
A!
}

This is an electronic reprint of the original article.

This reprint may differ from the original in pagination and typographic detail.

Kinnunen, Jami J.; Bruun, G.M.

\section{Induced interactions in a superfluid Bose-Fermi mixture}

Published in:

Physical Review A

DOI:

10.1103/PhysRevA.91.041605

Published: 01/01/2015

Document Version

Publisher's PDF, also known as Version of record

Please cite the original version:

Kinnunen, J. J., \& Bruun, G. M. (2015). Induced interactions in a superfluid Bose-Fermi mixture. Physical Review A, 91(4), 1-6. [041605]. https://doi.org/10.1103/PhysRevA.91.041605

This material is protected by copyright and other intellectual property rights, and duplication or sale of all or part of any of the repository collections is not permitted, except that material may be duplicated by you for your research use or educational purposes in electronic or print form. You must obtain permission for any other use. Electronic or print copies may not be offered, whether for sale or otherwise to anyone who is not an authorised user. 


\title{
Induced interactions in a superfluid Bose-Fermi mixture
}

\author{
J. J. Kinnunen \\ COMP Centre of Excellence and Department of Applied Physics, Aalto University, FI-00076 Aalto, Finland \\ G. M. Bruun \\ Department of Physics and Astronomy, Aarhus University, DK-8000 Aarhus C, Denmark
}

(Received 2 February 2015; published 30 April 2015)

\begin{abstract}
We analyze a Bose-Einstein condensate (BEC) mixed with a superfluid two-component Fermi gas in the whole BCS-BEC crossover. Using a quasiparticle random-phase approximation combined with Beliaev theory to describe the Fermi superfluid and the BEC, respectively, we show that the single-particle and collective excitations of the Fermi gas give rise to an induced interaction between the bosons, which varies strongly with momentum and frequency. It diverges at the sound mode of the Fermi superfluid, resulting in a sharp avoided crossing feature and a corresponding sign change of the interaction energy shift in the excitation spectrum of the BEC. In addition, the excitation of quasiparticles in the Fermi superfluid leads to damping of the excitations in the BEC. Besides studying induced interactions themselves, we can use these prominent effects to systematically probe the strongly interacting Fermi gas.
\end{abstract}

DOI: 10.1103/PhysRevA.91.041605

PACS number(s): 03.75.Kk, 67.85.De, 67.85.Pq

The interplay between induced interactions and superfluidity plays an important role in low-temperature physics. In metals, the phonon-mediated interaction between electrons leads to the formation of Cooper pairs [1], and induced electron-hole excitations significantly suppress the critical temperature of a BCS superconductor [2,3]. A prominent theory for high-temperature superconductivity is that it is caused by spin fluctuations leading to an attractive interaction [4], and induced interactions are important for understanding the properties of liquid-helium mixtures [5]. The systems where induced interactions are significant often consist of fermionic and bosonic degrees of freedom. In cold-atom gases, Bose-Fermi mixtures have been realized experimentally for sympathetic cooling [6-8], molecule formation [9-12], and studying few-body physics [13]. The theoretical studies have focused on mixtures where the Fermi gas is in the normal state [14-23]. Recently, an experimental breakthrough was reported with the realization of a mixture of superfluid ${ }^{7} \mathrm{Li}$ and ${ }^{6} \mathrm{Li}$ gases [24]. This opens up the exciting possibility to experimentally study the role of induced interactions in a Bose-Fermi mixture, where both components are superfluid.

Here we study a Bose-Einstein condensate (BEC) mixed with a two-component superfluid Fermi gas in the whole BCS-BEC crossover at zero temperature. Using a quasiparticle random-phase approximation (QRPA) to describe the excitations in the Fermi gas, combined with Beliaev theory for the bosons, we show how the fermions give rise to an induced frequency- or momentum-dependent Bose-Bose interaction, which diverges at the sound mode of the Fermi gas. This results in two qualitatively new effects. First, the dispersion relation of the bosons in the BEC is significantly changed at frequencies or momenta close to the sound mode of the Fermi gas. Second, bosonic excitations are damped due to dissipation, as they can excite quasiparticles in the superfluid Fermi gas [25]. These effects can be used to systematically probe the single-particle and collective properties of the strongly correlated Fermi gas.

We consider a gas of bosons with mass $m_{\mathrm{B}}$ mixed with a two-component $(\sigma=\uparrow, \downarrow)$ gas of fermions with mass $m_{\mathrm{F}}$. The populations of the two fermionic states are taken to be the same. The Hamiltonian of the Bose-Fermi mixture is $H=$ $H_{\mathrm{B}}+H_{\mathrm{F}}+H_{\mathrm{BF}}$, where

$$
H_{\mathrm{B}}=\sum_{\mathbf{k}} \epsilon_{k} a_{\mathbf{k}}^{\dagger} a_{\mathbf{k}}+\frac{1}{2 \mathcal{V}} \sum_{\mathbf{k}, \mathbf{k}^{\prime}, \mathbf{q}} V_{\mathrm{B}}(q) a_{\mathbf{k}+\mathbf{q}}^{\dagger} a_{\mathbf{k}^{\prime}-\mathbf{q}}^{\dagger} a_{\mathbf{k}^{\prime}} a_{\mathbf{k}}
$$

is the Bose Hamiltonian with $\epsilon_{k}=k^{2} / 2 m_{\mathrm{B}}$,

$$
H_{\mathrm{F}}=\sum_{\mathbf{k} \sigma} \frac{k^{2}}{2 m_{\mathrm{F}}} c_{\mathbf{k} \sigma}^{\dagger} c_{\mathbf{k} \sigma}+\frac{1}{\mathcal{V}} \sum_{\mathbf{k}, \mathbf{k}^{\prime}, \mathbf{q}} V_{\mathrm{F}}(q) c_{\mathbf{k}+\mathbf{q} \uparrow}^{\dagger} c_{\mathbf{k}^{\prime}-\mathbf{q} \downarrow}^{\dagger} c_{\mathbf{k}^{\prime} \downarrow} c_{\mathbf{k} \uparrow}
$$

is the Fermi Hamiltonian, and

$$
H_{\mathrm{BF}}=\frac{1}{\mathcal{V}} \sum_{\mathbf{k}, \mathbf{k}^{\prime}, \mathbf{q} \sigma} V_{\mathrm{BF}}(q) c_{\mathbf{k}+\mathbf{q} \sigma}^{\dagger} c_{\mathbf{k} \sigma} a_{\mathbf{k}^{\prime}-\mathbf{q}}^{\dagger} a_{\mathbf{k}^{\prime}}
$$

is the Bose-Fermi interaction. The operators $a_{\mathbf{k}}\left(c_{\mathbf{k} \sigma}\right)$ remove a boson (spin- $\sigma$ fermion) with momentum $\mathbf{k}, \mathcal{V}$ is the volume of the system, and we work in units where $\hbar=$ $k_{\mathrm{B}}=1$. In the following, we replace the interactions with the corresponding low-energy scattering matrices: $V_{\mathrm{B}}(q) \rightarrow \mathcal{T}_{\mathrm{B}}=$ $4 \pi a_{\mathrm{B}} / m_{\mathrm{B}}, \quad V_{\mathrm{F}}(q) \rightarrow \mathcal{T}_{\mathrm{F}}=4 \pi a_{\mathrm{F}} / m_{\mathrm{F}}$, and $V_{\mathrm{BF}}(q) \rightarrow \mathcal{T}_{\mathrm{BF}}=$ $2 \pi a_{\mathrm{BF}} / m_{\mathrm{r}}$, where $a_{\mathrm{B}}, a_{\mathrm{BF}}$, and $a_{\mathrm{F}}$ are the Bose-Bose, BoseFermi, and Fermi-Fermi scattering lengths, respectively, and $m_{\mathrm{r}}=m_{\mathrm{B}} m_{\mathrm{F}} /\left(m_{\mathrm{B}}+m_{\mathrm{F}}\right)$ is the reduced mass. As usual, this corresponds to summing all ladder diagrams in a vacuum.

The presence of the Fermi gas induces an effective interaction between the bosons since one boson tends to attract or repel fermions, giving rise to a local change in the fermion density, which is felt by the second boson. Combined with the direct Bose-Bose interaction, this results in total interaction

$$
V(q, \omega)=\mathcal{T}_{\mathrm{B}}+\mathcal{T}_{\mathrm{BF}}^{2} \chi(q, \omega) .
$$

Here $\chi(q, \omega)$ is the density-density response function for the fermions with momentum $\mathbf{q}$ and frequency $\omega$. The corresponding Feynman diagram for $V(q, \omega)$ is given in Fig. 1(a). The momentum dependence reflects the long range of the interaction, as density perturbations propagate in the Fermi gas. Similarly, the frequency dependence of the interaction 
(a)

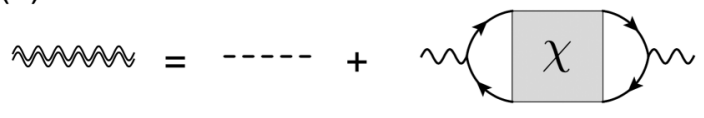

(b)

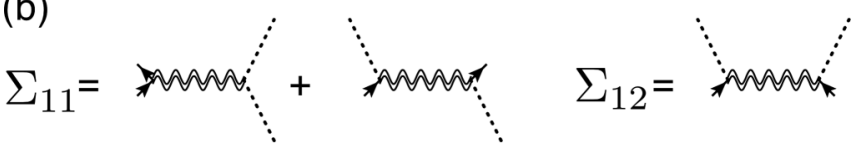

FIG. 1. (a) The effective interaction $V(q, \omega)$ (double wavy line) between the bosons. The dashed line is the bare Bose-Bose interaction $\mathcal{T}_{\mathrm{B}}$, single wavy lines are the Bose-Fermi interaction $\mathcal{T}_{\mathrm{BF}}$, and the solid lines are the Fermi Green's function. (b) The Bose self-energies $\Sigma_{11}(q, \omega)$ and $\Sigma_{12}(q, \omega)$. The dotted lines are excitations in and out of the BEC.

is due to the fact that it is not instantaneous since these perturbations have a finite speed.

In the weak-coupling BCS limit, $k_{\mathrm{F}} a_{\mathrm{F}} \rightarrow 0_{-}$, with $k_{\mathrm{F}}$ being the Fermi momentum of the Fermi gas, the density-density response function is given by

$$
\chi(q, \omega)=\left(\frac{v_{\mathrm{F}}}{c_{\mathrm{s}}}\right)^{2} \frac{\mathcal{N}\left(\epsilon_{\mathrm{F}}\right)}{3\left[\left(\frac{\omega}{c_{\mathrm{s}} q}\right)^{2}-1\right]}
$$

for frequency or momenta close to the AndersonBogoliubov sound mode $\omega=c_{\mathrm{s}} q$. The velocity is $c_{\mathrm{s}}=$ $v_{\mathrm{F}} \sqrt{1+2 k_{\mathrm{F}} a_{\mathrm{F}} / \pi} / \sqrt{3}$ [26-28], the density of states at the Fermi level $\epsilon_{\mathrm{F}}=k_{\mathrm{F}}^{2} / 2 m_{\mathrm{F}}$ is $\mathcal{N}\left(\epsilon_{\mathrm{F}}\right)=m_{\mathrm{F}} k_{\mathrm{F}} / \pi^{2}$, and $v_{\mathrm{F}}=$ $k_{\mathrm{F}} / m$. In the BEC regime, $k_{\mathrm{F}} a_{\mathrm{F}} \rightarrow 0_{+}$, the Fermi gas becomes a BEC consisting of diatomic molecules (dimers) with mass $2 m_{\mathrm{F}}$ and density $n_{\mathrm{F}} / 2$, where $n_{\mathrm{F}}=k_{\mathrm{F}}^{3} / 3 \pi^{2}$ is the total density of the fermions. The density-density response function is then, from Bogoliubov theory, given by [29]

$$
\chi(q, \omega)=\frac{n_{\mathrm{F}} q^{2}}{4 m_{\mathrm{F}}\left(\omega^{2}-\omega_{q}^{2}\right)} \simeq\left(\frac{v_{\mathrm{F}}}{c_{\mathrm{s}}}\right)^{2} \frac{\mathcal{N}\left(\epsilon_{\mathrm{F}}\right)}{12\left[\left(\frac{\omega}{c_{\mathrm{s}}}\right)^{2}-1\right]} .
$$

Here $\omega_{q}^{2}=q^{2}\left(4 m_{\mathrm{F}}\right)^{-1}\left(q^{2} / 4 m_{\mathrm{F}}+0.6 \mathcal{T}_{\mathrm{F}} n_{\mathrm{F}} / 2\right)$ is the Bogoliubov spectrum of the dimer BEC, where we have used the fact that the scattering length between the dimers is $0.6 a_{\mathrm{F}}$ in the BEC limit [30]. The second equality in (6) follows from the fact that $\omega_{q} \simeq c_{\mathrm{s}} q$ for small momenta, where $c_{\mathrm{s}}=$ $\sqrt{0.6 a_{\mathrm{F}} n_{\mathrm{F}} \pi / 2 m_{\mathrm{F}}^{2}}$ is the Bogoliubov sound speed.

In general, the density-density correlation function of the Fermi gas has a pole at $\omega=c_{\mathrm{s}} q$ in the whole BCS-BEC crossover, where $c_{\mathrm{s}}$ is the velocity of sound for a given scattering length $-\infty<a_{\mathrm{F}}<\infty$. It follows from (4) that the induced interaction between the bosons has the same pole structure: it is attractive for $\omega \leqslant c_{\mathrm{s}} q$, repulsive for $\omega \geqslant c_{\mathrm{s}} q$, and diverges when $\omega=c_{\mathrm{s}} q$. In addition, it has a nonzero imaginary part for frequency or momenta inside the quasiparticle continuum of the Fermi gas. It also follows from (4)-(6) that the strength $\kappa$ of the induced interaction scales as

$$
\kappa=\mathcal{T}_{\mathrm{BF}}^{2} \mathcal{N}\left(\epsilon_{\mathrm{F}}\right) \frac{v_{\mathrm{F}}^{2}}{c_{\mathrm{s}}^{2}},
$$

which should be compared with the strength $\mathcal{T}_{\mathrm{B}}$ of the direct Bose-Bose interaction.

We now examine the effects of the induced interaction on the excitation spectrum of the Bose gas. To this end, we need to calculate the density-density response function of the Fermi gas in the whole BCS-BEC regime. The density response function $\chi(1,2)$ is defined as a measure for how much the density of the Fermi gas changes at point (and time) 1 when a potential perturbation $\delta \phi$ is applied at point 2:

$$
\chi(1,2)=-\frac{\delta\langle n(1)\rangle}{\delta \phi(2)} .
$$

We apply a QRPA for calculating the Fourier transform of $\chi(1,2)$ in the superfluid state [28,31-34]. This is the simplest microscopic scheme which recovers the Anderson-Bogoliubov mode in the BCS regime and the Bogoliubov mode in the BEC regime. It yields a response function of the form

$$
\chi(q, \omega)=\frac{\chi_{0}(q, \omega)}{1-\mathcal{T}_{\mathrm{F}} L(q, \omega)},
$$

where $\chi_{0}(q, \omega)$ is a four-dimensional vector giving response due to quasiparticle excitations in the superfluid and $L$ is a $4 \times$ 4 matrix describing the couplings of the densities and the order parameter field. The collective modes manifest themselves as poles of the density response $\chi(q, \omega)$, i.e., as the zeros of the determinant,

$$
\operatorname{det}\left[1-\mathcal{T}_{\mathrm{F}} L(q, \omega)\right]=0 .
$$

The input parameters needed for the QRPA are the chemical potential $\mu$ and the pairing gap $\Delta$ of the Fermi superfluid, which are obtained self-consistently from BCS theory. We have for convergence added a small imaginary part $i \eta=i 10^{-3} \epsilon_{\mathrm{F}}$ to the frequencies and checked that the final numerical results do not depend on $\eta$, as long as $\eta \ll \epsilon_{\mathrm{F}}$. The details of this QRPA calculation can be found, for example, in Refs. [28,31].

Figure 2 shows the speed of the Anderson-Bogoliubov mode as a function of $1 / k_{F} a$, determined by finding the frequency $\omega$ at which the imaginary part of $\chi(q, \omega)$ is maximal for a given momentum $q$. The value of the momentum $q$ needs to be chosen small enough so that it probes the linear part of the collective mode branch. The speed of sound is then the slope

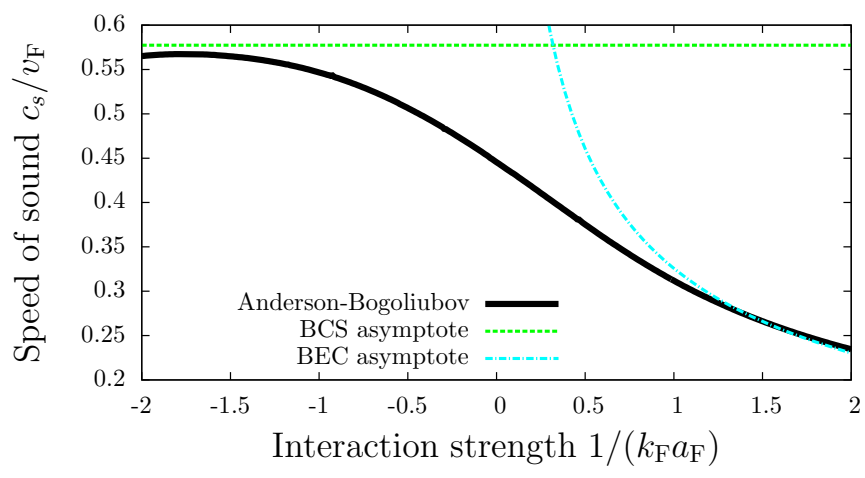

FIG. 2. (Color online) Speed of sound $c_{s}$ in the two-component Fermi superfluid as calculated from the pole of the density response function $\chi(\mathbf{q}, \omega)$. It approaches $c_{s}=v_{\mathrm{F}} / \sqrt{3}$ in the BCS limit (green dotted line) and the Bogoliubov result $c_{s}=v_{\mathrm{F}} \sqrt{k_{\mathrm{F}} a_{\mathrm{F}} / 3 \pi}$ in the BEC limit (blue dash-dotted curve). 


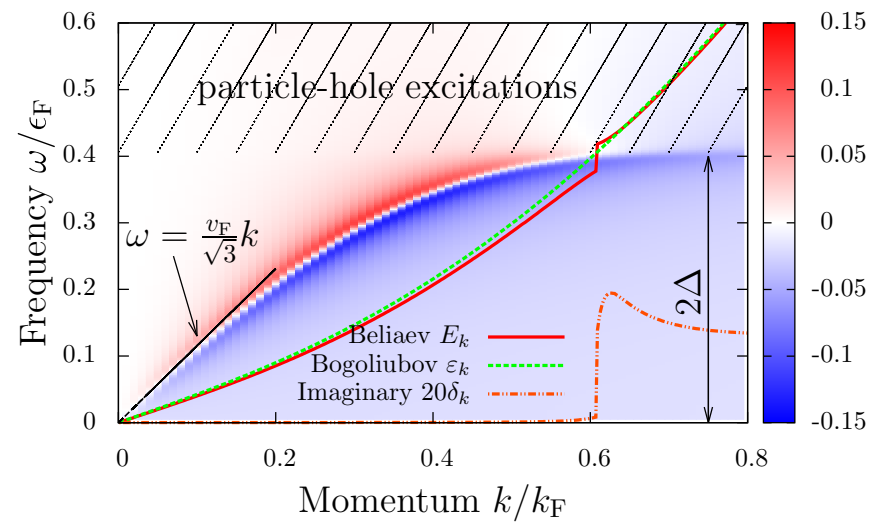

FIG. 3. (Color online) The blue (dark gray) and red (light gray) regions show $\operatorname{Re} \chi(q, \omega)$ for $k_{\mathrm{F}} a_{\mathrm{F}}=-1$. The black solid line is the weak-coupling Anderson-Bogoliubov mode, and the quasiparticle continuum for $\omega>2 \Delta$ is indicated by a dashed region. The green dashed line is the Bogoliubov spectrum $\varepsilon_{k}$ of the atomic BEC, and the red solid line is the Beliaev spectrum $E_{k}$ for the coupled Bose-Fermi mixture. The damping $\delta_{k}$ of the Beliaev excitations is shown as a red dash-dotted line.

$c_{s}=\omega / q$. In the BCS limit, the speed of sound approaches the weakly interacting limit $v_{\mathrm{F}} / \sqrt{3}$. The numerically calculated speed of sound deviates slightly from this in the very weakly interacting regime due to the difficulty of determining the slope when the pairing gap is very small. Our numerics reproduce to an excellent accuracy the speed of sound results in Ref. [35]. Note that this theory is, of course, not quantitatively correct in the whole BCS-BEC crossover. For instance, the speed of sound approaches $c_{s}=v_{\mathrm{F}} \sqrt{k_{\mathrm{F}} a_{\mathrm{F}} / 3 \pi}$ in the BEC limit (see Fig. 2). This corresponds to a molecular BEC with a scattering length $2 a_{\mathrm{F}}$ instead of the correct value $0.6 a_{\mathrm{F}}$. We emphasize, however, that the effects discussed below are completely general and do not depend on which approximate theory we apply to describe the strongly correlated system.

Figure 3 depicts the real part of the calculated densitydensity response for $k_{\mathrm{F}} a=-1$. At low frequency or momenta, we clearly see a sharp Anderson-Bogoliubov mode where $\operatorname{Re} \chi(\mathrm{q}, \omega)$ changes sign. The dispersion of this mode is close to the weak-coupling result $c_{\mathrm{s}}=v_{\mathrm{F}} / \sqrt{3}$. For higher momenta, the dispersion curves downwards when it approaches the quasiparticle continuum starting at energies above $2 \Delta$, with $\Delta \simeq 0.21 \epsilon_{\mathrm{F}}$.

Once $\chi(q, \omega)$ is calculated, we use Beliaev theory [36] to describe the effects of the resulting induced Bose-Bose interaction on the excitation spectrum of the atomic BEC. The single-particle propagator $\bar{G}(\mathbf{k}, \omega)$ for the BEC is a $2 \times 2$ matrix, and the Dyson equation reads

$$
\bar{G}(\mathbf{k}, \omega)=\bar{G}_{0}(\mathbf{k}, \omega)+\bar{G}_{0}(\mathbf{k}, \omega) \bar{\Sigma}(\mathbf{k}, \omega) \bar{G}(\mathbf{k}, \omega) .
$$

The bare propagator is

$$
\bar{G}_{0}(\mathbf{k}, \omega)=\left[\begin{array}{cc}
G_{0}(\mathbf{k}, \omega) & 0 \\
0 & G_{0}(\mathbf{k},-\omega)
\end{array}\right],
$$

and the self-energy is

$$
\bar{\Sigma}(\mathbf{k}, \omega)=\left[\begin{array}{cc}
\Sigma_{11}(\mathbf{k}, \omega) & \Sigma_{12}(\mathbf{k}, \omega) \\
\Sigma_{21}(\mathbf{k}, \omega) & \Sigma_{11}(\mathbf{k},-\omega)
\end{array}\right],
$$

where we have used the inversion symmetry $\mathbf{k} \leftrightarrow$ -k. The effects of interactions are included via the "Hartree-Fock" self-energies illustrated in Fig. 1(b), given by $\Sigma_{11}(\mathbf{k}, \omega)=\Sigma_{11}(\mathbf{k},-\omega)^{*}=n_{0} V(0,0)+n_{0} V(\mathbf{k}, \omega)$ and $\Sigma_{12}(\mathbf{k}, \omega)=\Sigma_{21}(\mathbf{k}, \omega)=n_{0} V(\mathbf{k}, \omega)$. Solving these equations for $\bar{G}(\mathbf{k}, \omega)$ yields the Green's functions for the diagonal elements,

$$
G(\mathbf{k}, \omega)=\frac{\omega+\epsilon_{k}+n_{0} V(\mathbf{k}, \omega)}{\omega^{2}-E(k, \omega)^{2}},
$$

where $E(k, \omega)=\epsilon_{k}^{2}+2 \epsilon_{k} n_{\mathrm{B}} V(k, \omega)$. The off-diagonal elements are $G_{12}(\mathbf{k}, \omega)=G_{21}(\mathbf{k}, \omega)=-n_{\mathrm{B}} V(\mathbf{k}, \omega) /\left[\omega^{2}-\right.$ $\left.E(k, \omega)^{2}\right]$, where $n_{\mathrm{B}}$ is the density of the BEC. The theory satisfies the Hugenholtz-Pines relation for the chemical potential $\mu=\Sigma_{11}(0)-\Sigma_{12}(0)=n_{\mathrm{B}} V(0,0)$. These interacting Green's functions describe excitations with energy dispersion $E_{k}$ given by solving

$$
E_{k}=\operatorname{Re} E\left(k, \omega=E_{k}\right) .
$$

In the absence of the induced interaction, this results in the usual Bogoliubov dispersion $\varepsilon_{k}=\sqrt{\epsilon_{k}^{2}+2 n_{\mathrm{B}} \mathcal{T}_{\mathrm{B}} \epsilon_{k}}$. However, due to the momentum and frequency dependence of $V(\mathbf{k}, \omega),(15)$ is implicit and needs to be solved numerically. The equation also yields damping of the excitations given by $\delta_{k}=\operatorname{Im} E\left(k, E_{k}\right)$.

Figures 3-5 show the dispersion $E_{k}$ obtained from (15), in the BCS $\left(k_{\mathrm{F}} a_{\mathrm{F}}=-1\right)$, unitarity $1 / k_{\mathrm{F}} a_{\mathrm{F}}=0$, and $\mathrm{BEC}$ $\left(k_{\mathrm{F}} a_{\mathrm{F}}=1\right)$ regimes of the Fermi gas, respectively. The calculations are performed using parameters corresponding to densities $n_{\mathrm{F}}=n_{\mathrm{B}}=10^{13} \mathrm{~cm}^{-3}$ and scattering lengths $a_{\mathrm{B}}=$ $a_{\mathrm{BF}}=400 a_{0}$, and inspired by the superfluid Bose-Fermi mixture experiment [24], we use the masses of ${ }^{6} \mathrm{Li}$ and ${ }^{7} \mathrm{Li}$ atoms. From (7), this yields $\kappa=4 \pi a_{\text {ind }} m_{\mathrm{B}}{ }^{-1} v_{\mathrm{F}}^{2} / c_{\mathrm{s}}^{2}$ for the strength of the induced interaction with the effective scattering length $a_{\text {ind }} \simeq 70 a_{0}$.

Consider first the BCS regime with $k_{\mathrm{F}} a_{\mathrm{F}}=-1$ shown in Fig. 3. Comparing the Bogoliubov spectrum $\epsilon_{k}$ for the atomic BEC decoupled from the Fermi gas with the Beliaev spectrum $E_{k}$ for the coupled Bose-Fermi mixture obtained from (15), we see that coupling to the Anderson-Bogoliubov mode results in an avoided crossing. Since we are neglecting backaction effects on the Fermi gas, this avoided crossing becomes a

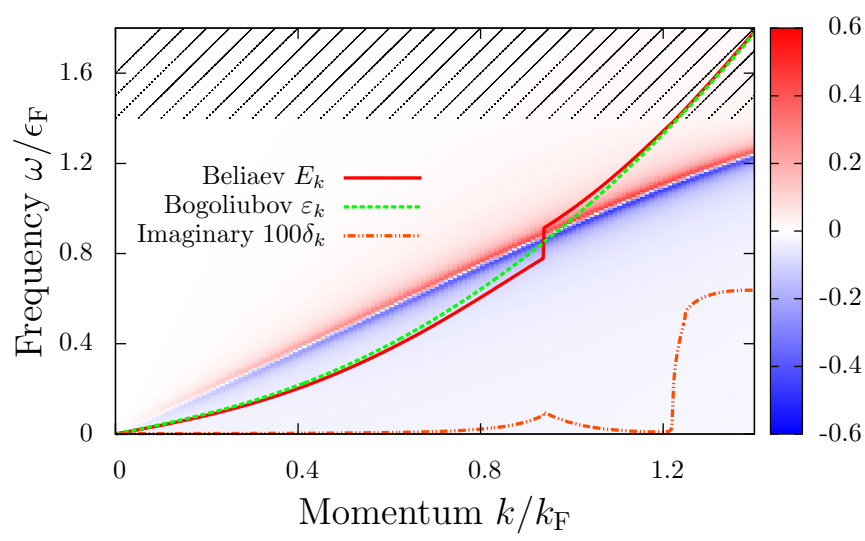

FIG. 4. (Color online) Same as Fig. 3, but for a unitary Fermi gas with $k_{\mathrm{F}} a_{\mathrm{F}}=\infty$. Here $\Delta \approx 0.69 E_{\mathrm{F}}$. 


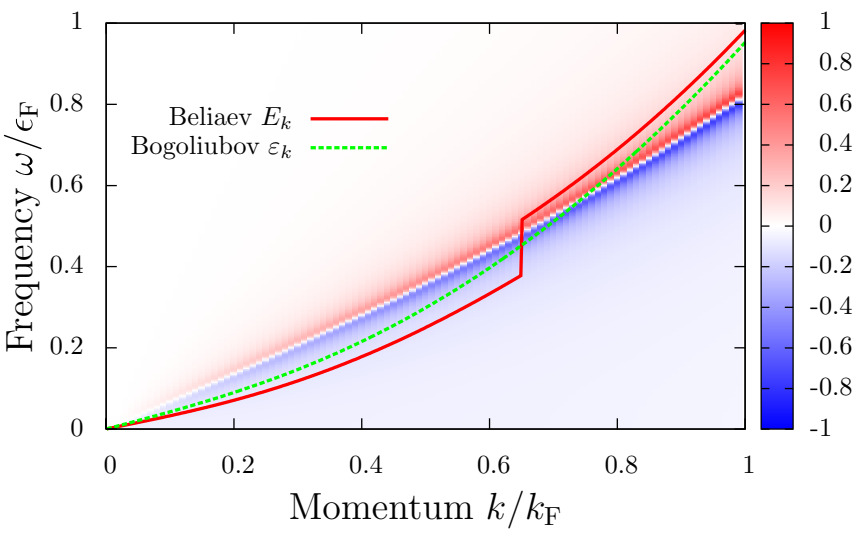

FIG. 5. (Color online) Same as Figs. 3 and 4, but for the Fermi superfluid in the $\mathrm{BEC}$ regime with $k_{\mathrm{F}} a_{\mathrm{F}}=1$. Here $\Delta \approx 1.35 \epsilon_{\mathrm{F}}$.

discontinuous jump in the bosonic excitation frequency. We expect this prediction to be qualitatively correct, except very close to the avoided crossing, since the induced interaction diverges when the two excitation frequencies are equal, making the corresponding avoided crossing sharp. Figure 3 also shows that the excitations of the BEC become damped when their energy is inside the quasiparticle continuum of the Fermi gas. This reflects that the excitation dissipates energy by exciting quasiparticles in the superfluid Fermi gas.

Figure 4 depicts the spectrum $E_{k}$ when the Fermi gas is in the unitarity regime with $1 / k_{\mathrm{F}} a_{\mathrm{F}}=0$. We again see that there is an avoided crossing, evidenced by a jump in the Beliaev dispersion $E_{k}$, when the Bogoliubov mode approaches the collective mode of the Fermi gas. In fact, the resulting energy shift is larger than in the BCS case since the spectral weight of the collective mode is larger in the unitarity regime. The bosonic excitations are again damped for energies $\omega>2 \Delta$. The small residual damping near the avoided crossing reflects, however, the small imaginary part i $\eta$ that we have built into the Fermi theory to obtain convergence. In the limit $\eta \rightarrow 0$, the bosonic excitations are undamped outside the quasiparticle continuum, even at the avoided crossing since it corresponds to the coupling of two undamped excitations.

Finally, Fig. 5 shows the dispersion $E_{k}$ in the BEC regime of the Fermi gas with $k_{\mathrm{F}} a_{\mathrm{F}}=1$. The avoided crossing feature and the energy shift in $E_{k}$ are now even more pronounced due to a smaller sound velocity of the Fermi gas, which approaches the Bogoliubov sound speed of a dimer BEC, thereby making $\kappa$ larger, as can be seen from (7). The quasiparticle continuum of the Fermi gas is outside the range of the plot due to the large pairing energy in the BEC regime. There is, therefore, no damping of the bosonic modes shown.

The above results show how the coupling between the superfluid bosons and fermions leads to significant effects on the spectrum of the atomic BEC, which depend on the properties of the Fermi gas. In the recent experiment on the superfluid ${ }^{6} \mathrm{Li}$ and ${ }^{7} \mathrm{Li}$ mixture, the Fermi-Fermi scattering length $a_{\mathrm{F}}$ could be tuned using a Feshbach resonance. We therefore plot in Fig. 6 the difference $E_{k}-\varepsilon_{k}$ between the Beliaev and Bogoliubov excitation spectra as a function of $a_{\mathrm{F}}$, keeping all other parameters as in Figs. 3-5. We also plot the damping of the mode. Two effects are apparent. First, since the

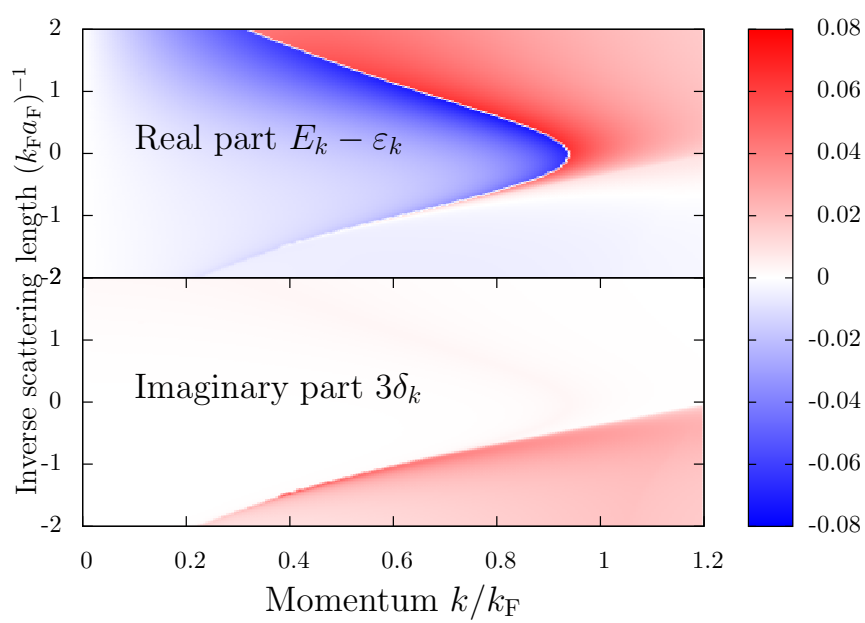

FIG. 6. (Color online) (top) Correction $\left(E_{k}-\varepsilon_{k}\right) / \epsilon_{\mathrm{F}}$ to the BEC dispersion due to the induced interaction as a function of Fermi-Fermi scattering length $k_{\mathrm{F}} a_{\mathrm{F}}$ and momentum $k$. (bottom) Decay $\delta_{k} / \epsilon_{\mathrm{F}}$ of the excitations in the BEC.

sound velocity in the Fermi gas depends on $a_{\mathrm{F}}$, the momentum where the bosonic mode exhibits the avoided crossing depends on $a_{\mathrm{F}}$. Also, the induced interaction in general decreases (increases) $E_{k}$ for energies below (above) the avoided crossing, as expected. The magnitude of the energy shift increases towards the BEC regime since spectral weight of the collective mode in the Fermi superfluid increases. Second, Fig. 6 clearly shows the damping caused by the coupling to the quasiparticle excitations of the superfluid Fermi gas. This quasiparticle continuum moves to higher momenta as the system approaches the BEC limit and the pairing gap increases. The residual damping below the quasiparticle continuum shown in Fig. 6 is, as explained above, a result of using a nonzero $\eta$ in the numerics, and it vanished for $\eta \rightarrow 0$. This illustrates how the collective and single-particle spectra of the strongly correlated Fermi gas can be mapped out by measuring its effects on the excitations in the BEC. We note that the effects can be increased significantly by increasing $a_{\mathrm{BF}}$ since $\kappa \propto a_{\mathrm{BF}}^{2}$. In addition to varying $a_{\mathrm{F}}$ and $a_{\mathrm{BF}}$, one can also vary $a_{\mathrm{B}}$, which will increase even further the ways one can probe the excitations in this Bose-Fermi mixture. The excitations of a $\mathrm{BEC}$ have already been measured using Bragg spectroscopy [37-44].

In conclusion, we examined a mixture of a $\mathrm{BEC}$ and a superfluid Fermi gas using Beliaev theory for the bosons combined with quasiparticle random-phase approximation for the fermions. The fermions were shown to mediate a frequency- or momentum-dependent interaction between the bosons, which leads to two qualitatively new effects. First, the induced interaction diverges at the sound mode of the Fermi gas, which results in a sharp avoided-crossing feature in the excitation spectrum of the BEC. Second, the excitation of quasiparticles in the Fermi gas leads to a damping of the excitations of the BEC. By varying the densities and scattering lengths of the system, these effects can be used to systematically probe the properties of the Fermi gas in the strongly correlated BCS-BEC crossover. Our work may be extended in a number of directions: It would be interesting to include the backaction of the bosons on the superfluid Fermi gas to obtain 
a detailed description of the avoided crossing of the sound modes. Trapping effects can be included using a local density approximation, which has proven to work well when considering short-wavelength Bragg scattering [39]. Finally, the theory can be extended to finite temperatures, which would result in a damping of BEC excitations for all momenta due to the presence of thermally excited quasiparticles in the Fermi gas.

\section{ACKNOWLEDGMENTS}

J.J.K. acknowledges support from the Academy of Finland through its Centres of Excellence Programme (2012-2017) under Project No. 251748. G.M.B. would like to acknowledge the support of the Hartmann Foundation via Grant No. A21352 and the Villum Foundation via Grant No. VKR023163.
[1] J. R. Schrieffer, Theory of Superconductivity (Perseus Books, New York, 1999).

[2] H. Heiselberg, C. J. Pethick, H. Smith, and L. Viverit, Influence of induced interactions on the superfluid transition in dilute Fermi gases, Phys. Rev. Lett. 85, 2418 (2000).

[3] L. P. Gor'kov and T. K. Melik-Barkhudarov, Contribution to the theory of superfluidity in an imperfect Fermi gas, Sov. Phys. JETP 13, 1018 (1961).

[4] D. J. Scalapino, The case for $d_{x^{2}-y^{2}}$ pairing in the cuprate superconductors, Phys. Rep. 250, 329 (1995).

[5] G. Baym and C. Pethick, Landau Fermi-Liquid Theory: Concepts and Applications (Wiley-VCH, Weinheim, 1991).

[6] A. G. Truscott, K. E. Strecker, W. I. McAlexander, G. B. Partridge, and R. G. Hulet, Observation of Fermi pressure in a gas of trapped atoms, Science 291, 2570 (2001).

[7] F. Schreck, L. Khaykovich, K. L. Corwin, G. Ferrari, T. Bourdel, J. Cubizolles, and C. Salomon, Quasipure Bose-Einstein condensate immersed in a Fermi sea, Phys. Rev. Lett. 87, 080403 (2001).

[8] G. Roati, F. Riboli, G. Modugno, and M. Inguscio, FermiBose quantum degenerate ${ }^{40} \mathrm{~K}-{ }^{87} \mathrm{Rb}$ mixture with attractive interaction, Phys. Rev. Lett. 89, 150403 (2002).

[9] C.-H. Wu, J. W. Park, P. Ahmadi, S. Will, and M. W. Zwierlein, Ultracold fermionic Feshbach molecules of ${ }^{23} \mathrm{Na}^{40} \mathrm{~K}$, Phys. Rev. Lett. 109, 085301 (2012).

[10] J. W. Park et al., Quantum degenerate Bose-Fermi mixture of chemically different atomic species with widely tunable interactions, Phys. Rev. A 85, 051602 (2012).

[11] M.-S. Heo et al., Formation of ultracold fermionic $\mathrm{NaLi}$ Feshbach molecules, Phys. Rev. A 86, 021602 (2012).

[12] T. D. Cumby, R. A. Shewmon, M. G. Hu, J. D. Perreault, and D. S. Jin, Feshbach-molecule formation in a Bose-Fermi mixture, Phys. Rev. A 87, 012703 (2013).

[13] S.-K. Tung, K. Jimenez-Garcia, J. Johansen, C. V. Parker, and C. Chin, Geometric scaling of Efimov states in a ${ }^{6} \mathrm{Li}^{-133} \mathrm{Cs}$ mixture, Phys. Rev. Lett. 113, 240402 (2014).

[14] L. Viverit, C. J. Pethick, and H. Smith, Zero-temperature phase diagram of binary boson-fermion mixtures, Phys. Rev. A 61, 053605 (2000).

[15] M. J. Bijlsma, B. A. Heringa, and H. T. C. Stoof, Phonon exchange in dilute Fermi-Bose mixtures: Tailoring the FermiFermi interaction, Phys. Rev. A 61, 053601 (2000).

[16] Z.-Q. Yu, S. Zhang, and H. Zhai, Stability condition of a strongly interacting boson-fermion mixture across an interspecies Feshbach resonance, Phys. Rev. A 83, 041603(R) (2011).

[17] D. Ludwig, S. Floerchinger, S. Moroz, and C. Wetterich, Quantum phase transition in Bose-Fermi mixtures, Phys. Rev. A 84, 033629 (2011).
[18] E. Fratini and P. Pieri, Mass imbalance effect in resonant BoseFermi mixtures, Phys. Rev. A 85, 063618 (2012).

[19] G. Bertaina, E. Fratini, S. Giorgini, and P. Pieri, Quantum Monte Carlo study of a resonant Bose-Fermi mixture, Phys. Rev. Lett. 110, 115303 (2013).

[20] T. Sogo, P. Schuck, and M. Urban, Bose-Fermi pairs in a mixture and the Luttinger theorem within a Nozières-Schmitt-Rink-like approach, Phys. Rev. A 88, 023613 (2013).

[21] J. H. Pixley, X. Li, and S. Das Sarma, Damping of long wavelength collective modes in spinor Bose-Fermi mixtures, arXiv: 1501.05015 .

[22] D. H. Santamore and E. Timmermans, Fermion-mediated interactions in a dilute Bose-Einstein condensate, Phys. Rev. A 78, 013619 (2008).

[23] A. P. Albus, S. A. Gardiner, F. Illuminati, and M. Wilkens, Quantum field theory of dilute homogeneous Bose-Fermi mixtures at zero temperature: General formalism and beyond mean-field corrections, Phys. Rev. A 65, 053607 (2002).

[24] I. Ferrier-Barbut, M. Delehaye, S. Laurent, A. T. Grier, M. Pierce, B. S. Rem, F. Chevy, and C. Salomon, A mixture of Bose and Fermi superfluids, Science 345, 1035 (2014).

[25] W. Zheng and H. Zhai, Quasiparticle lifetime in a mixture of Bose and Fermi superfluids, Phys. Rev. Lett. 113, 265304 (2014).

[26] P. W. Anderson, Random-phase approximation in the theory of superconductivity, Phys. Rev. 112, 1900 (1958).

[27] N. N. Bogoliubov, V. V. Tolmachev, and D. V. Shirkov, A New Method in the Theory of Superconductivity (Academy of Science, Moscow, 1958).

[28] A. Minguzzi, G. Ferrari, and Y. Castin, Dynamic structure factor of a superfluid Fermi gas, Eur. Phys. J. D 17, 49 (2001).

[29] P. Nozières and D. Pines, The Theory of Quantum Liquids (Perseus Books, New York, 1999), Vol. 2.

[30] D. S. Petrov, C. Salomon, and G. V. Shlyapnikov, Weakly bound dimers of fermionic atoms, Phys. Rev. Lett. 93, 090404 (2004).

[31] R. Côté and A. Griffin, Cooper-pair-condensate fluctuations and plasmons in layered superconductors, Phys. Rev. B 48, 10404 (1993).

[32] A. J. Leggett, Theory of a superfluid Fermi liquid. II. Collective oscillations, Phys. Rev. 147, 119 (1966).

[33] J. R. Engelbrecht, M. Randeria, and C. A. R. Sá de Melo, BCS to Bose crossover: Broken-symmetry state, Phys. Rev. B 55, 15153 (1997).

[34] G. M. Bruun and B. R. Mottelson, Low energy collective modes of a superfluid trapped atomic Fermi gas, Phys. Rev. Lett. 87, 270403 (2001).

[35] R. Combescot, M. Yu. Kagan, and S. Stringari, Collective mode of homogeneous superfluid Fermi gases in the BEC-BCS crossover, Phys. Rev. A 74, 042717 (2006). 


\section{J. J. KINNUNEN AND G. M. BRUUN}

[36] A. L. Fetter and J. D. Walecka, Quantum Theory of ManyParticle Systems (McGraw-Hill, New York, 1971).

[37] M. Kozuma et al., Coherent splitting of Bose-Einstein condensed atoms with optically induced Bragg diffraction, Phys. Rev. Lett. 82, 871 (1999).

[38] J. Stenger et al., Bragg spectroscopy of a Bose-Einstein condensate, Phys. Rev. Lett. 82, 4569 (1999).

[39] D. M. Stamper-Kurn et al., Excitation of phonons in a BoseEinstein condensate by light scattering, Phys. Rev. Lett. 83, 2876 (1999).

[40] J. Steinhauer, R. Ozeri, N. Katz, and N. Davidson, Excitation spectrum of a Bose-Einstein condensate, Phys. Rev. Lett. 88, 120407 (2002).
PHYSICAL REVIEW A 91, 041605(R) (2015)

[41] R. Ozeri, N. Katz, J. Steinhauer, and N. Davidson, Colloquium: Bulk Bogoliubov excitations in a Bose-Einstein condensate, Rev. Mod. Phys. 77, 187 (2005).

[42] S. B. Papp et al., Bragg spectroscopy of a strongly interacting ${ }^{85} \mathrm{Rb}$ Bose-Einstein condensate, Phys. Rev. Lett. 101, 135301 (2008).

[43] J. J. Kinnunen and M. J. Holland, Bragg spectroscopy of a strongly interacting Bose-Einstein condensate, New J. Phys. 11, 013030 (2009).

[44] S. Ronen, The dispersion relation of a Bose gas in the intermediate- and high-momentum regimes, J. Phys. B 42, 055301 (2009). 